Ml t i - obj ect i ve Net work Opport uni st i c Access for Group Mbbility in Mbbi I e I nt er net

\begin{tabular}{|l|l|}
\hline 著者 & TAO M ng, DONG M anxi ong, OTA Kaor u, HE Zhi mi n \\
\hline $\begin{array}{l}\text { j our nal or } \\
\text { publ i cat i on t i t l e }\end{array}$ & I EEE Syst ens Jour nal \\
\hline vol une & 12 \\
\hline number & 1 \\
\hline page $r$ ange & 10241033 \\
\hline year & 2018 03 \\
\hline URL & ht t p: //hdl . handl e. net /10258/00009987 \\
\hline
\end{tabular}




\title{
Multi-objective Network Opportunistic Access for Group Mobility in Mobile Internet
}

\author{
Ming Tao, Member, IEEE, Mianxiong Dong, Member, IEEE, Kaoru Ota, Member, IEEE, and Zhimin He
}

\begin{abstract}
The integration of existing and emerging heterogeneous wireless networks in Mobile Internet is a combination of diverse but complementary wireless access technologies. Satisfying a set of imperative constrains and optimization objectives, access network selection (ANS) for mobile node (MN) is an inherent procedure in mobility management that needs to be solved in a reasonable manner for the whole system to operate in an optimal fashion. However, ANS remains a significant challenge. Because many MNs with distinctive call characteristics are likely to have correlated mobility and may need to perform mobility management at the same time, this paper, with the goal of investigating group mobility solutions, proposes a Network Opportunistic Access for Group Mobility (NOA-GM) scheme. By analyzing the directional patterns of moving MNs and introducing the idea of opportunistic access, this scheme first identifies underloaded access networks as candidates. Then, the candidates are evaluated using normalized models of objective and subjective metrics. On this basis, the ANS problem for group mobility can be conducted as a multi-objective combination optimization and then transferred to a signal-objective model by considering the optimization of the performance of the whole system as a global goal while still achieving each MN's performance request. Using an improved Genetic Algorithm with newly designed evolutionary operators to solve the signal-objective model, an optimal result option for ANS for group mobility is achieved. Simulations conducted on the NS-2 platform show that NOA-GM outperforms the compared schemes in several critical performance metrics.
\end{abstract}

Index Terms-multi-objective, moving direction pattern, network opportunistic access, group mobility, genetic algorithm.

\section{INTRODUCTION}

Recently, the number of real-time mobile applications that require high data rates (such as mobile augmented reality) running on mobile devices has been growing rapidly. These applications need to be able to roam across internetworks to remain constantly connected, enabling mobile users to experience uninterrupted, anywhere, anytime services. Consequently, one important research issue lies in investigating

This work was supported in part by the National Natural Science Fund, China (Grant No. 61300198); the Guangdong University Scientific Innovation Project (No. 2013KJCX0177 \& No. 2014KTSCX188); the outstanding young teacher training program of the Education Department of Guangdong Province (YQ2015158); Guangdong Provincial Science \& Technology Plan Projects (No. 2016A010101035); JSPS KAKENHI (Grant No. 16K00117 \& No. 15K15976 \& No. 26730056), JSPS A3 Foresight Program.

Ming Tao is with the School of Computers, Dongguan University of Technology, Dongguan 523808, China (Email: ming.tao@mail.scut.edu.cn)

Mianxiong Dong (corresponding author) is with the Department of Information and Electronic Engineering, Muroran Institute of Technology, Japan (Email: mx.dong@csse.muroran-it.ac.jp)

Kaoru Ota is with the Department of Information and Electronic Engineering, Muroran Institute of Technology, Japan (Email: k.ota@ieee.org)

Zhimin $\mathrm{He}$ is with the College of Mathematics and Informatics, South China Agricultural University, Guangzhou 510642, China (Email: zmhe@scau.edu.cn) efficient and optimum solutions to handle IP mobility [1][8]. Generally, mobility management involves two operations: handoff management and location management. The former may be divided into three phases on the time dimension: i) Network Discovery, ii) Handoff Determination and iii) Handoff Execution. During the Network Discovery phase, information concerning the networks available for access can be obtained by performing a port scan. The Handoff Determination phase then selects the next network to access for the roaming $\mathrm{MN}$ and decides when (or whether) to perform the handoff. Based on these decisions, the Handoff Execution phase completes the specified handoff procedures. Therefore, access network selection (ANS) is an inherent procedure in handoff management that needs to be solved in a reasonable manner to preserve the quality of experience (QoE) for mobile users and improve the performance of the whole system.

Selecting an optimal access network for a single $\mathrm{MN}$ is already a well-investigated research area. Traditionally, studies have assumed that there is only one choice for access technology; therefore, ANS has been performed based on channel quality as indicated by the received signal strength (RSS) or other measurements and the available resources in the access networks (e.g., bandwidth). However, an ANS decision made by only a single criterion (e.g., RSS) may be not the optimal one. Recently, with the development of mobile wireless technology, the prevalent mobile Internet has been a combination of diverse but complementary access technologies. Such a mobile communication environment with complex network coverage, access networks with heterogeneous access technologies and service abilities, MNs with distinct call characteristics, and running applications with different Quality of Service (QoS) requirements have all raised new challenges for the ANS problem.

In this paper, group mobility is considered a group-based behavior because many MNs have distinct call characteristics but are likely to have correlated mobility and may perform mobility management at the same time. Rather than focusing only on achieving individual performance of a single $\mathrm{MN}$, ANS for group mobility is investigated here to satisfy the respective access requests and operate across the entire system in an optimal fashion. The scenario of ANS for group mobility discussed here can be illustrated as follows. Assuming that $\mathrm{MN}=\left\{\mathrm{MN}_{1}, \mathrm{MN}_{2}, \ldots, \mathrm{MN}_{i}, \ldots, \mathrm{MN}_{m}\right\}$ is a group of mobile nodes with distinct mobility and call characteristics and $\mathrm{AN}=\left\{\mathrm{AN}_{1}, \mathrm{AN}_{2}, \ldots, \mathrm{AN}_{j}, \ldots, \mathrm{AN}_{n}\right\}$ is a set of heterogeneous access networks, the final results of ANS for group mobility can be expressed as a $0-1$ matrix $A=\left\{a_{i j}\right\}_{m \times n}$. Because a $\mathrm{MN}$ is served by only one access network at a time, 
if $i$-th $\mathrm{MN}$ is served by $j$-th access network, $a_{i j}=1$; otherwise, $a_{i j}=0$. To address this issue, this paper proposes a Network Opportunistic Access scheme for Group Mobility (NOA-GM) that makes the following contributions.

1. By considering the directional patterns in which MNs are moving, the access networks along those paths all have the opportunity to be visited. By introducing the idea of opportunistic access, only underloaded networks can be selected as candidates. This reduces the probability of handoff dropping and new call blocking.

2. To evaluate the candidate networks, both objective (Application QoS and Network Conditions) and subjective (User Preferences) evaluation metrics are considered to achieve the tradeoff, respectively, to formulate a normalized model. In the evaluation system for these metrics, membership functions are employed to unify the value directions of the involved evaluation attributes.

3. Based on the formulations of the evaluation metrics, the addressed ANS for group mobility is conducted as a multiobjective combination optimization. By making the goal of optimizing the whole system performance while maintaining each MN's performance request, the multi-objective optimization is transferred to a signal-objective model. To achieve unity between the subjective preference and the objective authenticity of ANS decisions, the theory of information entropy is used to balance the weights of the evaluation metrics.

4. The stated ANS problem with many imperative constraints and optimization objectives has been proven to be NP-hard; therefore, an improved genetic algorithm with newly designed evolutionary operators and good convergence is developed to solve this problem in a reasonable fashion and achieve an optimal result.

The remainder of this paper is organized as follows. Section 2 discusses the current related research achievements. Section 3 thoroughly investigates the scheme of multi-objective network opportunistic access. Section 4 introduces an improved genetic algorithm to solve the addressed problem. Based on elaborately designed experiments, Section 5 investigates the performance of NOA-GM compared to available alternatives. Section 6 summarizes and concludes this paper.

\section{RELATED WORK}

To provide mobility services that have better robustness and scalability, the mobile Internet employs a multilevel hierarchical architecture in which the lower layers in the hierarchy consist of high-bandwidth wireless cells with small coverage areas, and the upper layers consist of cells that provide lower bandwidth but a much wider coverage area. MNs equipped with multiple wireless interfaces can access these networks on the move. Because each node must follow a specified process to perform binding updates [9], this hierarchical architecture causes additional signal and packet processing overhead. Therefore, it is necessary to find an optimal hierarchy to minimize the overall cost. By investigating different network parameters, such as transmission/update costs and sessionto-mobility (SMR), there are some analytical models for determining the optimal hierarchy. With such hierarchical and complex coverage, determining the best access network for a roaming $\mathrm{MN}$ in a visited area is a critical issue that involves improving system performance. This topic has received much attention over the past few decades. The proposed methods can be broadly divided into two categories: static and adaptive.

As a representative static scheme, distance-based selection often chooses server network access in terms of distance criteria, mobility patterns (e.g. velocity), and so on [10]. For example, the upper layer access network has wider coverage and is particularly suitable for MNs undergoing frequent handoffs to reduce the cost of global binding updates, while the lower layer access network is preferable for slower-moving MNs to reduce the packet transmission cost. Because the mobility state of an MN may vary over time, defining precise and universal thresholds that clearly distinguish MN mobility patterns is still critical.

The alternative adaptive scheme usually considers factors such as traffic loads, mobility patterns, service characteristics and so on. In the distance-based ANS with dynamic load control (DMS-DLC) mechanisms proposed by Kusin et al. [11], a current load-based preference value for an access network is first defined. Then, this mechanism makes ANS decisions using distance criteria and the preference value. However, because the load option for achieving dynamic load control is continually updated, coordination among access networks requires additional signaling overhead. In contrast, the mobility-based load control (MLC) proposed by Pack et al. [12], integrates a threshold-based admission control algorithm with an SMR-based replacement algorithm to adaptively mitigate the burden of accessing a network.

Additionally, due to the imperative constraints and desired optimal goals, ANS also can be conducted as an NP-hard problem. Both game theory and heuristic algorithms have attracted significant attention as solutions to this problem. By considering factors such as the MN mobility pattern, network load status, and call request preference, Liou et al. [13] propose a bargaining game-based ANS. Using a Fuzzy Set Representation TOPSIS (FSR-Utility-TOPSIS) method to resolve the inconsistencies of conflicting decision criteria, Chamodrakas et al. [14] attempt to optimize ANS decisions with parameterized utility functions using multiple criteria. Feng et al. [15] propose an evolutionary game model to describe the dynamics of ANS and used a reinforcement learning algorithm based on evolutionary equilibrium as the solution. By formulating ANS as a stochastic game with negative network externality and modeling the operation of achieving the optimal decision as a multidimensional Markov Decision Process, Yang et al. [16] propose a modified value iteration algorithm to obtain an ANS decision. Ge et al. [17] formulate ANS as a restless bandit problem and solve it using a first-order relaxation-based primal-dual index heuristic algorithm. By taking the efficient resource management of different networks as an optimization problem and transferring the problem into a good selection of weights to match their QoS parameters, Maria et al. [18] develop several heuristic methods using simple rules to find the best available network. By considering varying channel contention and transmission data rates, Cheung et al. [19] formulate ANS as a finite-horizon 
sequential decision problem and solve it using a dynamic programming (DP)-based optimal random access algorithm.

While acknowledging these proposals in the literature, which have been found to be efficient, each still has some limitations and works effectively only in certain specific cases. In the emerging integrated hybrid wireless network environment, ANS can be further optimized by taking into account additional suitable access networks that can provide roaming MNs with better mobile services. Additionally, this paper focuses on ANS for group mobility, which raises new challenges in operating the whole system in an optimal fashion while maintaining each MN's performance request.

\section{Multi-ObJective Networks Opportunistic ACCESS}

\section{A. Determining Candidate Access Networks Determination Based on Moving Direction Patterns}

In practical scenarios, most end users follow regular routines during business hours and might do similar things within a geographical area, so the mobility profile of an $\mathrm{MN}$ is typically regular to a certain extent [20], [21]. In terms of a mobility profile, the moving direction, denoted as $\theta$, can be obtained from GPS location data. These data are used as the major metric to predict the moving direction pattern for an MN. Assuming that the MN can move in any direction with equal probability, the probability density function of $\theta$ can be represented as $f(\theta)=1 / 2 \pi,(-\pi<\theta<\pi)$.

By considering the moving direction pattern of an $\mathrm{MN}$, it is logical that access networks along the moving direction all have the opportunity to be visited. To reduce the handoff dropping probability and new call blocking probability, the idea of opportunistic access is introduced, in which only underloaded access networks can be chosen as the candidates. To reflect the differences and the underlying communication strength of access networks, the load intensity, denoted as $\operatorname{Load}[i]$, is normalized in (1) [9], and a threshold $\rho$ is employed to divide the load status into two types: Normal and Overload.

$$
\begin{aligned}
& \operatorname{Load}[i] \\
& =\left\{\begin{array}{l}
1, \text { if }\left(q_{i}=Q_{i} \text { or } s_{i} \geq S_{i} \text { or } k_{i} \geq K_{i} \| \operatorname{Load}[i] \geq \rho\right) \\
w_{1} \frac{q_{i}}{Q_{i}}+w_{2} \frac{s_{i}}{S_{i}}+w_{3} \frac{k_{i}}{K_{i}}, \text { others, }\left(\sum_{j=1}^{3} w_{j}=1\right)
\end{array}\right.
\end{aligned}
$$

For the $i$-th access network, $q_{i}$ and $Q_{i}$ are the current queue length and maximum queue length, respectively, $s_{i}$ and $S_{i}$ are the gained session arrival rate in the latest period and the maximum session arrival rate within the capacity, $k_{i}$ and $K_{i}$ are the number of currently served MNs and the maximum number of served MNs within the capacity. The normalized values are $q_{i} / Q_{i}, s_{i} / S_{i}$ and $k_{i} / K_{i}$ within $[0,1]$. The transmission ability can be obtained by $q_{i} / Q_{i}$, while the strength of underlying communications can be obtained by $s_{i} / S_{i}$ and $k_{i} / K_{i}$. In addition, the weighted factors $w_{j}(j=1,2,3)$ are introduced. These weighted factors can be adjusted dynamically to achieve the expected focus. Because the three parameters have the

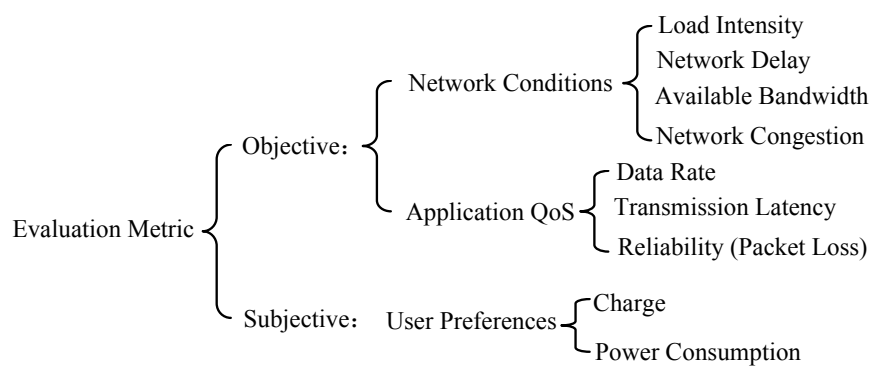

Fig. 1. The evaluation system employed for the candidates.

same value direction, which affects the load evaluation, the evaluated effect is consistent no matter how it is adjusted.

Based on these stated definitions, access networks periodically evaluate their load status using the dynamic weighted load evaluation algorithm implementation shown in Algorithm 1. To avoid further aggravating the workload of overloaded networks, only underloaded access networks should admit a handoff or take new call requests.

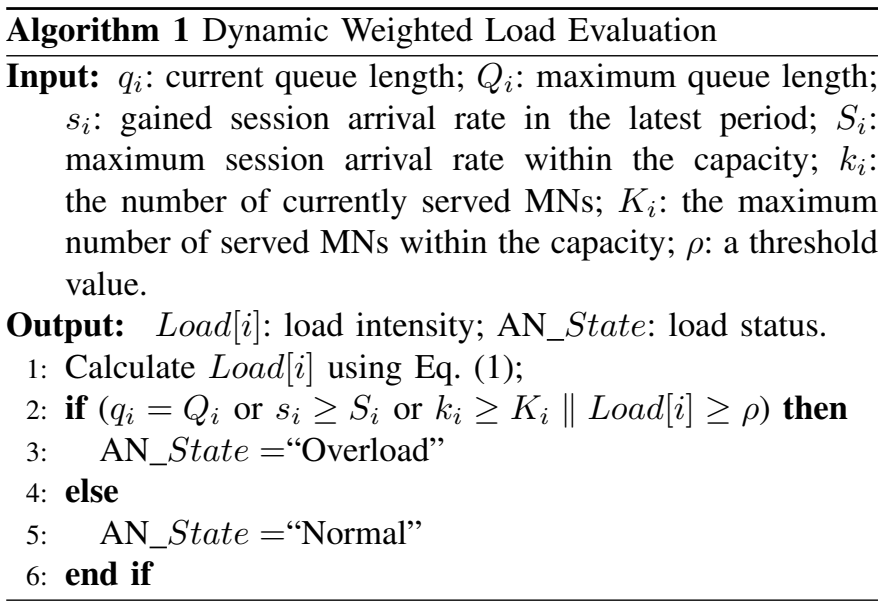

\section{B. Formulations of Evaluation Metrics}

To achieve an optimal result option that maximizes the whole system performance while maintaining each MN's performance, the evaluation system employed for the candidates is shown in Fig. 1, which includes three dimensions of objective and subjective metrics. In the following formulations of the evaluation metrics, the symbols used along with their corresponding explanations are listed in Table. I.

Because of intensive resource competition for network resources or conflicts, an ongoing handoff process for an MN might be dropped. Similarly, if the current service capability of the target access network is beyond its limit, a new mobile call request might be blocked due to the lack of adequate resources. Thus, using the available network information is beneficial for achieving a balanced load across different networks and can also relieve congestion in some cases. To evaluate the network conditions, evaluation attributes such as load intensity, network delay, available bandwidth, and network congestion are considered in this system. The normalized evaluation 
TABLE I

LIST OF THE USED SYMBOLS.

\begin{tabular}{|c|c|}
\hline Symbol & Explanation \\
\hline$N L_{j}^{i}, N D_{j}^{i}, B W_{j}^{i}, N C_{j}^{i}$ & $\begin{array}{l}\text { Current load intensity, network delay, available bandwidth, and network congestion of the } j \text { - } \\
\text { th access network when the } i \text {-th MN selects the } j \text {-th access network as the serving network, } \\
\text { respectively. }\end{array}$ \\
\hline$N L_{j}, N D_{j}, B W_{j}, N C_{j}$ & $\begin{array}{l}\text { Acceptable load intensity, network delay, available bandwidth, and network congestion of the } \\
j \text {-th access network, respectively. }\end{array}$ \\
\hline$N L_{\max }, N D_{\max }, N C_{\max }, B W_{\max }$ & $\begin{array}{l}\text { Acceptable maximum load intensity, network delay, network congestion, and available band- } \\
\text { width of the candidate access network, respectively. }\end{array}$ \\
\hline$N L_{\min }, N D_{\min }, N C_{\min }, B W_{\min }$ & $\begin{array}{l}\text { Acceptable minimum load intensity, network delay, network congestion, and available band- } \\
\text { width of the candidate access network, respectively. }\end{array}$ \\
\hline$D R_{i \text {-actual }}^{j}, T L_{i \text {-actual }}^{j}, P L_{i \text {-actual }}^{j}$ & $\begin{array}{l}\text { Actual data rate, transmission latency, and packet loss when the } i \text {-th MN selects the } j \text {-th access } \\
\text { network as the serving network, respectively. }\end{array}$ \\
\hline$D R_{i \text {-expect }}, T L_{i \text {-accept }}, P L_{i \text {-accept }}$ & Expected data rate, acceptable transmission latency and packet loss of the $i$-th $\mathrm{MN}$, respectively. \\
\hline$D R_{\max }, T L_{\max }, P L_{\max }$ & $\begin{array}{l}\text { Maximum data rate, transmission latency, and packet loss of the candidate access network, } \\
\text { respectively. }\end{array}$ \\
\hline$D R_{\min }, T L_{\min }, P L_{\min }$ & $\begin{array}{l}\text { Minimum data rate, transmission latency, and packet loss of the candidate access network, } \\
\text { respectively. }\end{array}$ \\
\hline$C_{i \text {-actual }}^{j}, E_{i \text {-actual }}^{j}$ & $\begin{array}{l}\text { Actual charge, battery power consumption when the } i \text {-th } \mathrm{MN} \text { selects the } j \text {-th access network } \\
\text { as the serving network, respectively. }\end{array}$ \\
\hline$C_{i \text {-budget }}, E_{\text {i-accept }}$ & Budget and acceptable battery power consumption of the $i$-th MN, respectively. \\
\hline$C_{\max }, E_{\max }$ & Maximum charge, and battery power consumption of the candidate access network, respectively. \\
\hline$C_{\min }, E_{\min }$ & Minimum charge, and battery power consumption of the candidate access network, respectively. \\
\hline
\end{tabular}

Condition (net) $[i, j]=\alpha_{1} \cdot$ Load $+\alpha_{2} \cdot$ Delay $+\alpha_{3} \cdot$ Bandwidth $+\alpha_{4} \cdot$ Congestion

$$
\begin{aligned}
& =\alpha_{1} \cdot \frac{\left|N L_{j}^{i}-N L_{j}\right|}{\max \left\{N L_{\max }-N L_{j}, N L_{j}-N L_{\min }\right\}}+\alpha_{2} \cdot \frac{\left|N D_{j}^{i}-N D_{j}\right|}{\max \left\{N D_{\max }-N D_{j}, N D_{j}-N D_{\min }\right\}} \\
& \quad+\alpha_{3} \cdot \frac{\left|B W_{j}^{i}-B W_{j}\right|}{\max \left\{B W_{\max }-B W_{j}, B W_{j}-B W_{\min }\right\}}+\alpha_{4} \cdot \frac{\left|N C_{j}^{i}-N C_{j}\right|}{\max \left\{N C_{\max }-N C_{j}, N C_{j}-N C_{\min }\right\}} \\
& \text { s.t. } \quad \sum_{i=1}^{4} \alpha_{i}=1,0<\alpha_{i}<1
\end{aligned}
$$

model is defined in (2), where $\alpha_{i}$ is the influence weight of each evaluation attribute.

$$
\begin{aligned}
& Q o S(\operatorname{app})[i, j] \\
& =\beta_{1} \cdot \text { Rate }+\beta_{2} \cdot \text { Latency }+\beta_{3} \cdot \text { Loss } \\
& =\beta_{1} \cdot \frac{\left|D R_{i \text {-actual }}^{j}-D R_{\text {i-expect }}\right|}{\max \left\{D R_{\max }-D R_{\text {i-expect }}, D R_{\text {i-expect }}-D R_{\min }\right\}} \\
& \quad+\beta_{2} \cdot \frac{\left|T L_{i \text {-actual }}^{j}-T L_{\text {-accept }}\right|}{\max \left\{T L_{\max }-T L_{i \text {-accept }}, T L_{\text {i-accept }}-T L_{\min }\right\}} \\
& \quad+\beta_{3} \cdot \frac{\left|P L_{i \text {-actual }}^{j}-P L_{\text {-accept }}\right|}{\max \left\{P L_{\max }-P L_{i-\text { accept }}, P L_{\text {-accept }}-P L_{\min }\right\}} \\
& \text { s.t. } \quad \sum_{i=1}^{3} \beta_{i}=1,0<\beta_{i}<1
\end{aligned}
$$

Different applications running on MNs have different QoS requirements. The system for evaluating Application QoS considers evaluation attributes such as data rate, transmission latency, reliability, and (packet losses). The normalized QoS evaluation model is defined in (3), where $\beta_{i}$ is the influence weight of each evaluation attribute.

In group mobility, different users have distinct preferences. User preferences can be used to broker special requests that prioritize one access network over another. The system for evaluating user preferences considers attributes such as charge and battery power consumption. Charge is a major consideration for users because different network operators use different billing strategies. Variations in billing plans may influence user access choice. Battery power is also a significant factor that can affect access in some cases. When the battery level is low, users may prefer to access networks that require lower power consumption. The normalized evaluation model is defined in (4), where, $\gamma_{i}$ is the influence weight assigned to each evaluation attribute. 


$$
\begin{aligned}
& \text { Preference(user) }[i, j] \\
& =\gamma_{1} \cdot \text { Charge }+\gamma_{2} \cdot \text { Energy } \\
& =\gamma_{1} \cdot \frac{\left|C_{i \text {-actual }}^{j}-C_{i \text {-budget }}\right|}{\max \left\{C_{\max }-C_{i \text {-budget }}, C_{i \text {-budget }}-C_{\min }\right\}} \\
& \quad+\gamma_{2} \cdot \frac{\left|E_{i \text {-actual }}^{j}-E_{\text {-accept }}\right|}{\max \left\{E_{\max }-E_{i \text {-accept }}, E_{i \text {-accept }}-E_{\min }\right\}} \\
& \text { s.t. } \quad \sum_{j=1}^{2} \gamma_{j}=1,0<\gamma_{j}<1
\end{aligned}
$$

\section{Optimization Objective Model and Weight Assignment}

In the introduced metrics evaluation system, the evaluation attributes can be classified into benefit and cost ones. The value direction of the former is in accordance with the overall goal, but the latter has the opposite value direction.

In the network conditions evaluation system, when the $i$-th MN selects the $j$-th access network as the serving network, the overall goal is to minimize the performance impact imposed on the target access network. Specifically, after the selection, when the load intensity is heavy, network delays are high, or network congestion is significant, the performance impact will also be significant. Similarly, low available bandwidth will also have a serious impact on performance. Hence, the first three evaluation attributes hence are related to costs, and the available bandwidth is the benefit attribute. In the application QoS evaluation system, the overall goal is to enhance the user's QoS experience in the target access network. To that end, when the data rate is higher, the QoS experience would be better, but reduced transmission delay and fewer packets lost would also result in a better QoS experience. Hence, the data rate is the benefit attribute, while transmission delay and fewer packets are the cost attributes. In the user preferences evaluation system, the overall goal is to maximize the user's preference when selecting the target access network. The user's preference is greater when the resulting charge and battery power consumption are smaller; hence, both these evaluation attributes are cost attributes.

The membership functions are used to unify the value directions of the evaluation attributes with the overall goal in the respective evaluation system [22]. Because the considered evolution attributes have been normalized In the stated formulations and the value ranges have been linearly transferred into $[0,1]$, the benefit attributes do not need to be processed further, but the cost attributes must be processed by using the simple membership function shown in (5).

$$
y^{\prime}=1-y, 0 \leq y \leq 1
$$

Accordingly, as defined in (6), the addressed ANS can be conducted as a multi-objective combination optimization, where, $x_{i j}$ is a decision variable. If the $i$-th MN selects the $j$-th access network as the serving network then $x_{i j}=1$; otherwise, $x_{i j}=0$.

For group mobility, by optimizing the whole system performance as the global goal while maintaining each MN's performance, the multi-objective optimization can be transferred to the signal-objective model as shown in (7), where $\omega_{k}$ represents the introduced weights.

$$
\begin{aligned}
& \max \text { Performance(system) } \\
& \begin{array}{r}
=\sum_{i}^{n} \sum_{j}^{m} \omega_{1} \cdot \text { Condition }(\text { net })[i, j]+\omega_{2} \cdot \operatorname{QoS}(\mathrm{app})[i, j] \\
\quad+\omega_{3} \cdot \operatorname{Preference}(\text { user })[i, j]
\end{array} \\
& \text { s.t. } \quad \sum_{k=1}^{3} \omega_{k}=1, \quad 0<\omega_{k}<1, k=1,2,3
\end{aligned}
$$

After formulating the optimization objection function, determining the weight value for each evaluation metric is the most important and difficult problem. Because the network conditions and application QoS are objective evaluation metrics, while user preference is a subjective one, to achieve unity between the subjective preference and the objective authenticity of ANS decisions, the theory of information entropy is used to evenly determine the assigned weights.

Assuming that $\Theta_{j k}(j=1,2, \ldots, n ; k=1,2,3)$ is the performance of the $k$-th evaluation metric in the $j$-th access network, then $p_{j k}=\Theta_{j k} / \sum_{j=1}^{n} \Theta_{j k}$. According to the theory of information entropy, the entropy of the $k$-th evaluation metric is $E_{k}=-(\ln n)^{-1} \sum_{j=1}^{n} p_{j k} \ln p_{j k}$, where the formula for the $\operatorname{logarithm} \ln n$ is used to facilitate the normalization. Moreover, if $p_{j k}=0$, then $p_{j k} \ln p_{j k}=0$. Therefore, the weight of the $k$-th evaluation metric can be defined in (8).

$$
\omega_{k}=\left(1-E_{k}\right) / \sum_{k=1}^{3}\left(1-E_{k}\right)
$$

As stated above, the final result option of ANS for group mobility can be represented as a $0-1$ matrix $A=\left\{a_{i j}\right\}_{m \times n}$. Accordingly, for each result option, there will be a corresponding value of Performance(system). By sorting the value of Performance(system), the option corresponding to the maximal Performance(system) would be the one finally adopted.

\section{Algorithm Design And Analysis}

Generally, when the perceived QoE in an MN's current network has deteriorated drastically, the ANS decision must be made in a timely manner, so that the roaming $\mathrm{MN}$ can connect to a new access network and enjoy improved QoE. To solve the stated ANS problem effectively (which has been proven to be NP-hard), an Improved Genetic Algorithm (IGA) with newly designed evolutionary operators is developed in Algorithm 2. A new modular arithmetic based crossover operator is designed to expand the search scope; therefore, IGA can create offspring by using the modulus operator to turn the sums of corresponding values in the two parent individuals into the remainder. Additionally, a uniform mutation operator is designed to enhance population diversity and prevent the algorithm from reaching the local optimum. Assume that $X=\left\{x_{1}, x_{2}, \ldots, x_{n}\right\}$ is a parent individual participating in the mutation, and in turn, randomly generating a $x_{i}^{\prime}$ from $[1, m]$ 


$$
\left.\begin{array}{rl}
\max \text { Condition (net) } & =\sum_{i=1}^{n} \sum_{j=1}^{m} x_{i j} \cdot \text { Condition }(\text { net })[i, j] \\
& =\sum_{i=1}^{n} \sum_{j=1}^{m} x_{i j} \cdot\left[\alpha_{1} \cdot(1-\text { Load })+\alpha_{2} \cdot(1-\text { Delay })+\alpha_{3} \cdot \text { Bandwidth }+\alpha_{4} \cdot(1-\text { Congestion })\right] \\
\max Q o S(\mathrm{app}) & =\sum_{i}^{n} \sum_{j}^{m} x_{i j} \cdot \operatorname{QoS}(\mathrm{app})[i, j]=\sum_{j}^{m} \sum_{i}^{n} x_{i j} \cdot\left[\beta_{1} \cdot \text { Rate }+\beta_{2} \cdot(1-\text { Latency })+\beta_{3} \cdot(1-\text { Loss })\right] \\
\max \text { Preference (user }) & =\sum_{i}^{n} \sum_{j}^{m} x_{i j} \cdot \operatorname{Preference}(\text { user })[i, j]=\sum_{j}^{m} \sum_{i}^{n} x_{i j} \cdot\left[\gamma_{1} \cdot(1-\text { Cost })+\gamma_{2} \cdot(1-\text { Energy })\right]
\end{array}\right\}
$$

with equal probability of replacing $x_{i}$. Then, a new individual $X^{\prime}=\left\{x_{1}^{\prime}, x_{2}^{\prime}, \ldots, x_{n}^{\prime}\right\}$ as a mutated offspring is obtained.

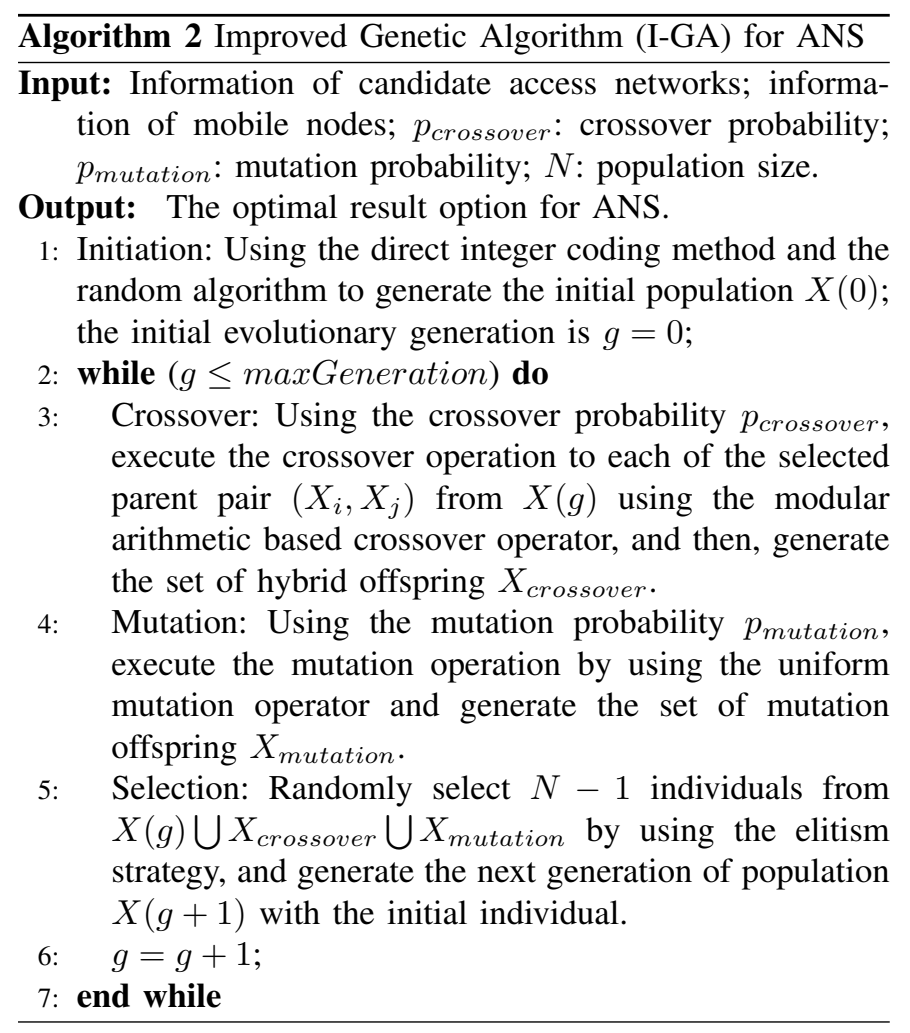

To demonstrate the effectiveness of I-GA, the algorithm convergence is discussed as follows. First, by using the elitism strategy in the selection operation, the population generations of $\{X(0), X(1), \ldots, X(g), \ldots\}$ in the feasible region of I-GA are monotonous, so the relationship of the fitness functions of the two adjacent generations can be achieved in (9).

$$
\forall g, \min \{f(x) \mid x \in X(g)\} \leq \min \{f(x) \mid x \in X(g+1)\}
$$

Assuming that $X_{a}$ and $X_{c}$ are any two individuals in the generations of $X(g)$ and $X\left(g^{\prime}\right)$ then $\left(g^{\prime}>g\right)$. In terms of the stated assumptions, the probability of selecting $X_{a}$ as the crossover individual is $p_{\text {crossover }}>0$. If $X_{b}$ is either of the hybrid offspring of $X_{a}$, the probability of selecting $X_{b}$ as the mutation individual is $p_{\text {mutation }}>0$. After the crossover and mutation operations, the probability of generating $X_{c}$ from $X_{a}$ can be represented as shown in (10).

$$
p\left(X_{a} \underset{\text { mutation }}{\stackrel{\text { crossover }}{\longrightarrow}} X_{c}\right) \geq p_{\text {crossover }} \cdot p_{\text {mutation }} \cdot p\left(X_{b} \stackrel{\text { mutation }}{\longrightarrow} X_{c}\right)
$$

Assuming that $X_{b}=\left\{x_{b}^{1}, x_{b}^{2}, \ldots, x_{b}^{n}\right\}$ and $X_{c}=\left\{x_{c}^{1}, x_{c}^{2}, \ldots\right.$, $\left.x_{c}^{n}\right\}$, in terms of the designed uniform mutation operator, the probability of mutating $x_{b}^{i}$ into $x_{c}^{i}$ is $\frac{1}{m-1}$, and the probability of generating $X_{c}$ from $X_{b}$ can therefore be defined as shown in (11). Next, (10) can be reformulated as (12). Finally, we can conclude that $X_{c}$ can be generated from $X_{a}$ by performing finite crossover and mutation operations. Assuming $\varepsilon(\varepsilon>0)$ is a positive constant, the distance between $X(g)$ and $X\left(g^{\prime}\right)$ is denoted as $d\left[X(g), X\left(g^{\prime}\right)\right]$; therefore, it is smaller than $\varepsilon$, i.e. $d\left[X(g), X\left(g^{\prime}\right)\right]<\varepsilon$, and the population generations of $\{X(0), X(1), \ldots, X(g), \ldots\}$ can be taken as a bounded Cauchy sequence in which, when $g^{\prime}>g \rightarrow \infty, d\left[X(g), X\left(g^{\prime}\right)\right] \rightarrow$ 0 . Accordingly, the stated theoretical analyses show that the proposed I-GA would converge to the global optimal solution with a probability of 1 .

$$
\begin{gathered}
p\left(X_{b} \stackrel{\text { mutation }}{\longrightarrow} X_{c}\right)=\frac{1}{(m-1)^{n}}>0 \\
p\left(X_{a} \underset{\text { mutation }}{\stackrel{\text { crossover }}{\longrightarrow}} X_{c}\right)=p_{\text {crossover }} \cdot p_{\text {mutation }} \cdot \frac{1}{(m-1)^{n}}>0
\end{gathered}
$$

\section{Simulation And Analysis}

\section{A. Simulation Setup}

With the NS-2 platform, Fig. 2 is an illustration of the used hierarchical network topology, in which WCDMA cellular systems fully cover the service area, but OFDMA-based WMAN cells and randomly deployed IEEE 802.11 WLANs provide only partial coverage. Note that, to avoid the boundary effects perceived by the roaming $\mathrm{MN}$, a wraparound model is used as well. The significant simulation parameters are configured in Table. II.

During system initialization, $100 \mathrm{MNs}$ are evenly distributed in this scenario and they randomly select the serving access network. Subsequently, during each simulation interval 


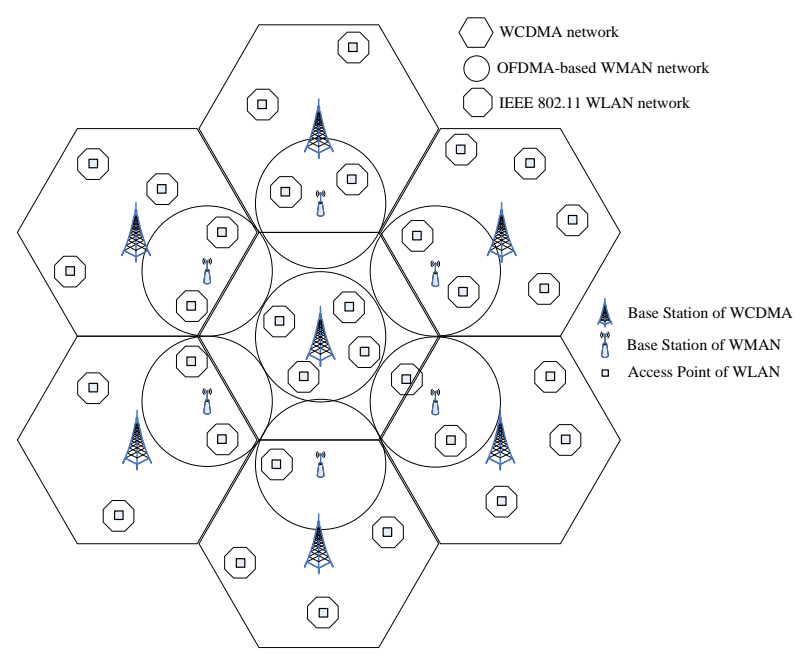

Fig. 2. Illustration of the used network topology.

TABLE II

THE CONFIGURATIONS OF SIGNIFICANT PARAMETERS IN THE SIMULATIONS.

\begin{tabular}{cccc}
\hline \hline Parameters (unit) & WCDMA & WMAN & WLAN \\
\hline Cell radius (km) & 2 & 1.6 & 0.1 \\
Frame/time slot duration (ms) & 10 & 5 & $9 \times 10^{-3}$ \\
Carrier frequency (GHz) & 2 & 2.5 & 2.4 \\
Bandwidth (Mbps) & 5 & 22 & 54 \\
Delay (ms) & 200 & 100 & 50 \\
Energy consumption in active state (W) & 1.2 & 3.5 & 4.5 \\
Charge & 1 & 0.5 & 0.2 \\
\hline \hline
\end{tabular}

$(\tau=100 \mathrm{~s})$, new mobile calls arrivals are regarded as a stochastic process conforming to a Poisson distribution. Note that each deployed MN may have distinct mobility and call characteristics; the mobility profiles are obtained by using the classical Random Waypoint model, and MN velocity $(\mathrm{km} / \mathrm{h})$ is a random variable uniformly distributed in $[1,50]$. The session arrival rates of the applications running on the MNs follow a Poisson distribution based on the parameter $\lambda$, and $\lambda$ is randomly distributed in $[1,10]$. The QoS requirements for typical applications are listed in Table. III.

TABLE III

QOS REQUIREMENTS FOR TYPICAL APPLICATIONS.

\begin{tabular}{ccccccc}
\hline \hline $\begin{array}{c}\text { QoS } \\
\text { requirements }\end{array}$ & $\begin{array}{c}D R_{\min } \\
(\mathrm{kbps})\end{array}$ & $\begin{array}{c}D R_{\max } \\
(\mathrm{kbps})\end{array}$ & $\begin{array}{c}T L_{\min } \\
(\mathrm{ms})\end{array}$ & $\begin{array}{c}T L_{\max } \\
(\mathrm{ms})\end{array}$ & $\begin{array}{c}P L_{\min } \\
(\%)\end{array}$ & $\begin{array}{c}P L_{\max } \\
(\%)\end{array}$ \\
\hline Voice & 32 & 64 & 75 & 150 & 0.01 & 1 \\
Web Browsing & 128 & 1000 & 250 & 500 & 1 & 5 \\
Video Streaming & 128 & 1000 & 2000 & 4000 & 1 & 5 \\
Video Conference & 512 & 5000 & 75 & 150 & 1 & 5 \\
\hline \hline
\end{tabular}

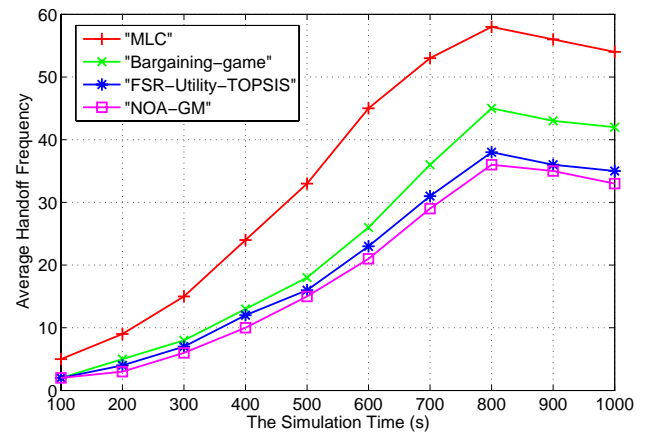

Fig. 3. Average handoff frequency.

\section{B. Comparison and Analysis}

We conducted a simulation analysis to demonstrate the average performance of handoff frequency, load balancing among the hierarchies, average handoff latency, handoff dropping probability, new call blocking probability and the average throughput of NOA-GM. We selected three representative ANS schemes, MLC [10], the bargaining game-based scheme [11] and the FSR-Utility-TOPSIS [18] using the same simulation settings for a fair comparative study.

The average handoff frequency (AHF) is defined as the average number of ongoing calls changing from one served access network to another during the simulation. Fig. 3 shows the AHF performance. A handoff may be caused by the following reasons: an MN moves to the cell edge, the capacity of the current serving access network drops below a predefined threshold, or the selected serving access network is not the optimal available network. From Fig. 3, we can see that AHF increases as the simulation continues. When the system service capability limits are exceeded, the increased probability of handoff dropping will reduce the AHF. Comparatively, by passively migrating the burden among the access networks to achieve a dynamic load balance, MLC has a higher AHF. Because the number of deployed WLANs is relatively greater and the load of each WLAN is relatively lighter on average, an MN using MLC would like to select a WLAN as its serving access network. With the introduced dwelling factors, an MN using the bargaining game-based scheme always selects the access network with large dwelling factors, and the MNs experience relatively fewer handoffs during the simulation. FSR-UtilityTOPSIS resolves the inconsistencies in the conflicting decision criteria, and NOA-GM comprehensively considers the tradeoff between the objective and the subjective criteria. The decisions to change the serving access network in these two schemes are more rational, and the average handoff frequencies are more balanced than those of the other two schemes. Accordingly, for load balancing performance among the hierarchies shown in Fig. 4, NOA-GM and FSR-Utility-TOPSIS outperform the other two schemes as well. Note that the load in each hierarchy is evaluated in total without having to evaluate the specific load in each cell.

Due to the delay in transmitting registration information, the relationships of handoff latencies among the heterogeneous networks can be discussed as follows. Generally, the 


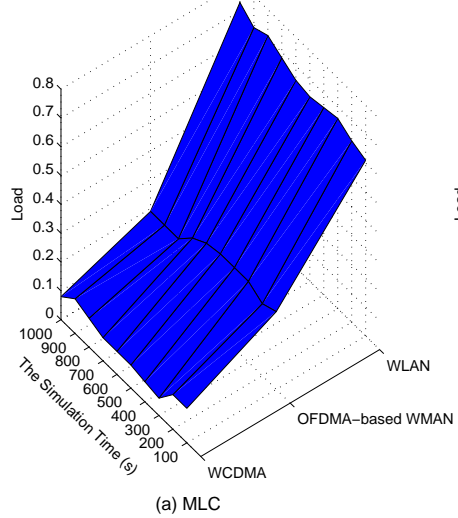

Fig. 4. Load balance.

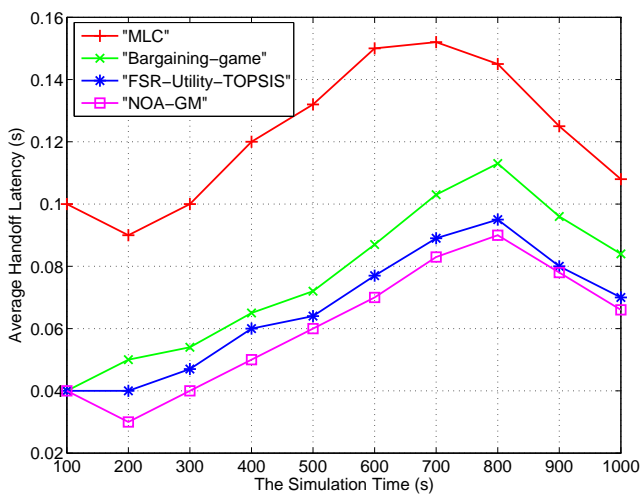

Fig. 5. Average handoff latency.

handoff latency from a bottom network to a top network is longer than that from top to bottom, $L($ Bottom $\rightarrow$ Top $)>$ $L(T o p \rightarrow B o t t o m)$. In the simulation setup in this paper, $L(W L A N \rightarrow W C D M A)>L(W M A N \rightarrow W C D M A)>$ $L(W L A N \rightarrow W M A N)$, and $L(W C D M A \rightarrow W L A N)>$ $L(W C D M A \rightarrow W M A N)>L(W M A N \rightarrow W L A N)$. Accordingly, we discuss the performance on average handoff latency (AHL) which is defined as $\operatorname{SUM}(L) / N$. $\operatorname{SUM}(L)$ is the sum of triggered handoff latencies and $N$ is the number of MNs. Similarly, by comparative analysis of the characteristics of the four compared schemes discussed for AHF, as shown in Fig. 5, we can clearly observe that the AHL of NOA-GM has a distinct advantage.

The handoff dropping probability (HDP) is defined as the probability that an existing mobile call fails to handoff to the target access network. The performance of the schemes on HDP is shown in Fig. 6. Generally, if the target access network is overloaded, the handoff request would be dropped immediately. In the bargaining game-based scheme, a candidate access network allocates more resources to the MN with larger dwelling factors. If the load intensity is beyond the predefined threshold or the remaining capacity cannot satisfy the handoff request, the handoff would be dropped immediately. In particular, because existing calls in the target

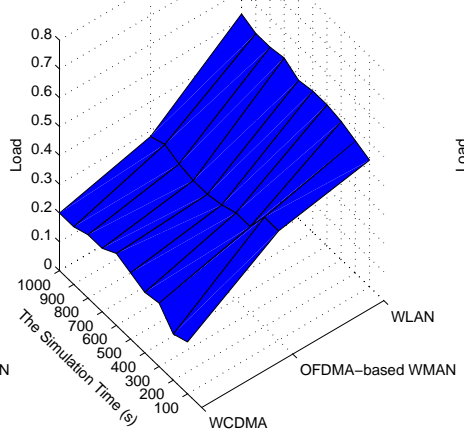

(c) FSR-Utility-TOPSIS

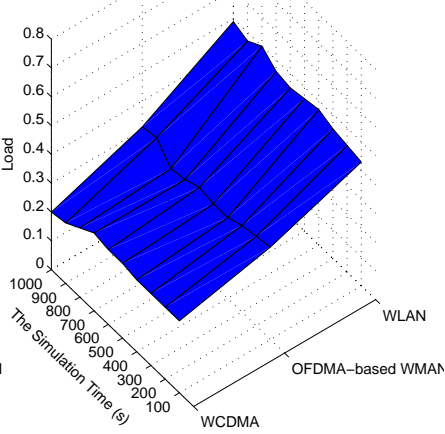

(d) NOA-GM

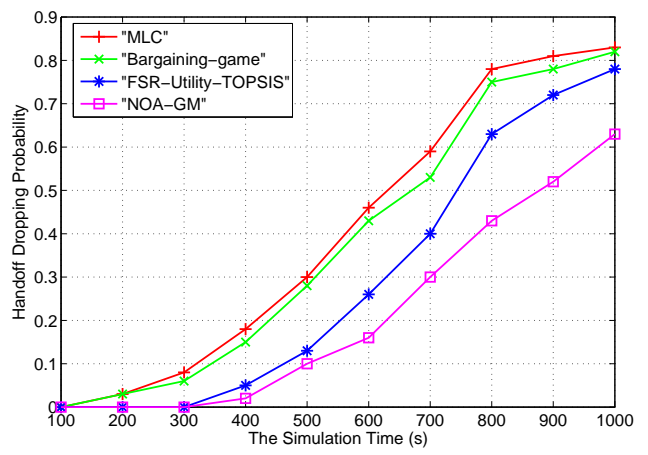

Fig. 6. Handoff dropping probability.

access network are only passively migrated when the load intensity is high, MLC will have a higher probability of dropping the handoff due to insufficient capacity. FSR-UtilityTOPSIS still calculates the overall rating of the networks for a handoff request, but the highest ranking network may not be an underloaded one. Therefore, when the candidates are overload, the handoff may be dropped. In NOA-GM, rather than considering only a single handoff request, the global goal is to maximize the entire system performance. Additionally, only underloaded access networks along the moving direction of MN are chosen as the candidates. Therefore, the HDP of NOA-GM is superior to the HDPs of the compared schemes.

New call block probability (CBP) is defined as the probability that a new mobile call will fail to be admitted by the target access network. Fig. 7 shows the performance of the schemes on CBP. Similarly, if the target access network is overloaded, the new call request would be blocked. In general, all new calls prefer WLANs over the other two types of access networks. Initially, because there are relatively more deployed WLANs and each WLAN is relatively lighter on average, the CBP is close to zero. However, as the number of served calls increases and MN mobility increase, the competition for these finite resources becomes more serious. If there is not enough capacity in the target access network, the bargaining game-based scheme will block the new call. Only if there 


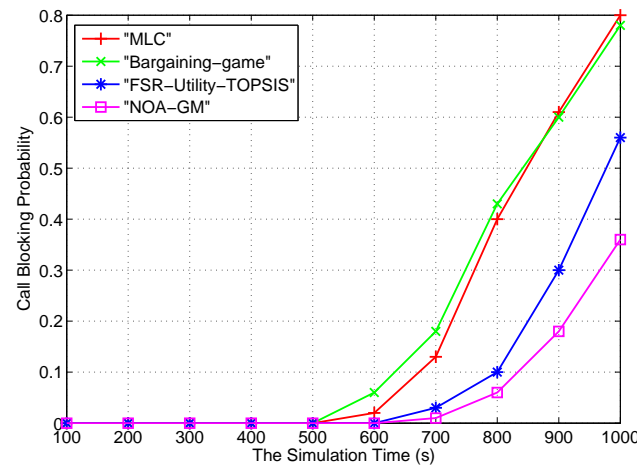

Fig. 7. Call blocking probability.

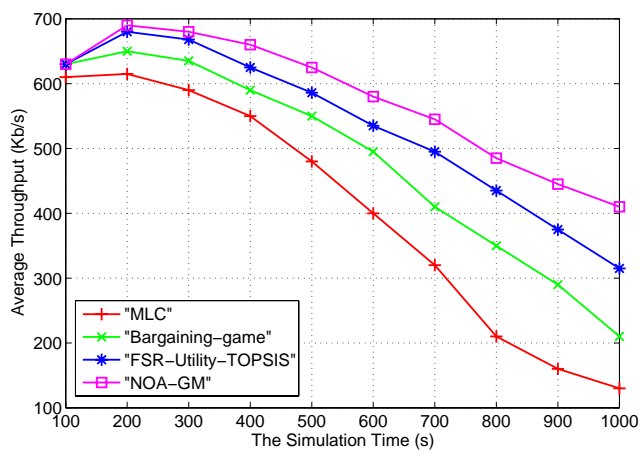

Fig. 8. Average throughput.

is an existing call with a higher SMR in the target access network, would MLC admit the new call by replacing the existing one. Moreover, the highest ranking network may not be an underloaded one in FSR-Utility-TOPSIS; therefore, the new call might be blocked when the system is saturated. Because NOA-GM introduces the idea of opportunistic access to determine the candidate access network, the CBP In NOAGM is smaller than the CBPs of the compared schemes.

Average throughput (AT) is defined as the average ratio of received valid packets of all MNs during a unit of time. Fig. 8 shows the performance of the schemes on AT. As the simulation continues, the increased number of mobile calls session first enhances the average system throughput. However, as the served calls and MN mobility continue to increase, the throughput begins to decline. For example, the throughput in MLC is seriously influenced by communication interruptions caused by higher handoff frequencies. Although the bargaining game-based scheme and FSR-Utility-TOPSIS have fewer or more balanced handoff frequencies, the contention for resources and communication collisions also cause the throughput to deteriorate. Specifically, the increased HDP and $\mathrm{CBP}$ are the main factors that cause the throughput to deteriorate. Comparatively, NOA-GM outperforms the other schemes due to its advantages on AHF, HDP and CBP.

\section{CONCLuSiON}

Roaming across heterogeneous wireless networks constitutes a challenge when providing continuity services for mo- bile users. ANS as an important operation because handoff decisions directly influence system performance and the end users' experiences. In this paper, a network opportunistic access scheme (NOA-GM) is proposed to investigate the issue of ANS for group mobility, in which many MNs with distinct call characteristics are likely to have correlated mobility and may perform mobility management at the same time. By analyzing the mobility direction patterns and introducing the idea of opportunistic access, only underloaded access networks along the moving direction of MNs are selected as candidate networks. The three dimensions of objective and subjective evaluation metrics are then introduced for these candidates, and normalized evaluation models are formulated. To unify the value directions of the involved evaluation attributes in the respective evaluation system, a membership function is used as well. Subsequently, the ANS problem is conducted as a multi-objective combination optimization. By adopting optimization of the entire system performance as the global goal while still preserving each MN's performance, the conducted multi-objective optimization is transferred to a signalobjective model, and the theory of information entropy is used to determine balanced weights for the evaluation metrics. Finally, an improved genetic algorithm with newly designed evolutionary operators and good convergence is developed to solve this problem. The simulations conducted on the NS-2 platform show that NOA-GM is superior to compared schemes on several critical performance metrics.

The ANS problem addressed in this paper is only the first significant problem in handoff decisions. After determining the target access network, further decisions concerning when (or whether) to execute the handoff trigger and when to perform the handoff execution must be made as well [23]. Due to the randomness of human mobility, the handoff trigger might be imperative or unnecessary. Failing to detect an imperative handoff trigger or a false alarm from an unnecessary handoff trigger will cause mobile users' QoE to deteriorate badly. Therefore, developing an intelligent and reliable handoff trigger scheme to avoid performance degradation due to missed trigger detections and false alarms remains an interesting problem that we hope to further explore in future work.

\section{REFERENCES}

[1] F. Siddiqui and S. Zeadally, "Mobility management across hybrid wireless networks: Trends and challenges," Computer Communications, vol. 29, no. 9, pp. 1363-1385, 2006.

[2] R. Angelo, P. Samuel, and K. Suresh, "Secure route optimization for mipv6 using enhanced cga and dnssec," IEEE Systems Journal, vol. 7, no. 3, pp. 351-362, 2013.

[3] H. Zhu, M. Dong, S. Chang, Y. Zhu, M. Li, and X. Shen, "Zoom: Scaling the mobility for fast opportunistic forwarding in vehicular networks," in Proceedings of 32nd IEEE International Conference on Computer Communications (INFOCOM), 2013, pp. 2832-2840.

[4] V. Berrocal-Plaza, M. A. Vega-Rodríguez, and J. M. Sánchez-Pérez, "Optimizing the mobility management task in networks of four world capital cities," Journal of Network and Computer Applications, vol. 51, pp. 18-28, 2015.

[5] K. Ota, M. Dong, Z. Cheng, L. X. Wang, Junbo, and X. Shen, "Oracle: Mobility control in wireless sensor and actor networks," Computer Communications, vol. 35, no. 9, pp. 1029-1037, 2012.

[6] G. Fabio, C. Luca, and B. Carlos J, "Distributed mobility management for future 5g networks: overview and analysis of existing approaches,' IEEE Communications Magazine, vol. 53, no. 1, pp. 142-149, 2015. 
[7] S. Guo, M. Dong, G. Minyi, and L. Chen, "A comparative analysis of mobility-based lifetime-aware multicast routing protocols for manets," in Proceedings of IPSJ International Conference on Mobile Computing and Ubiquitous Networking, 2008, pp. 64-71.

[8] M. Tao, H. Yuan, X. Hong, and J. Zhang, "Smartho: mobility pattern recognition assisted intelligent handoff in wireless overlay networks," Soft Computing, vol. online, no. DOI 10.1007/s00500-015-1747-9, pp. $1-10,2015$.

[9] M. Tao, H. Yuan, and W. Wei, "Active overload prevention based adaptive map selection in hmipv6 networks," Wireless networks, vol. 20, no. 2, pp. 197-208, 2014.

[10] E. Natalizio, A. Scicchitano, and S. Marano, "Mobility anchor point selection based on user mobility in hmipv6 integrated with fast handover mechanism." in Proceedings of IEEE Wireless Communications and Networking Conference (WCNC), 2005, pp. 1434-1439.

[11] Z. Kusin and M. S. Zakaria, "Dynamic load control mechanism in hierarchical mipv6," in Proceedings of International Conference on Electrical Engineering and Informatics (ICEEI). IEEE, 2011, pp. 1-5.

[12] S. Pack, T. Kwon, and Y. Choi, "A mobility-based load control scheme in hierarchical mobile ipv6 networks," Wireless Networks, vol. 16, no. 2, pp. 545-558, 2010.

[13] Y.-S. Liou, R.-H. Gau, and C.-J. Chang, "A bargaining game based access network selection scheme for hetnet," in Proceedings of IEEE International Conference on Communications (ICC). IEEE, 2014, pp. 4888-4893.

[14] I. Chamodrakas and D. Martakos, "A utility-based fuzzy topsis method for energy efficient network selection in heterogeneous wireless networks," Applied Soft Computing, vol. 11, no. 4, pp. 3734-3743, 2011.

[15] Z. Feng, L. Song, Z. Han, D. Niyato, and X. Zhao, "Cell selection in two-tier femtocell networks with open/closed access using evolutionary game," in Proceedings of IEEE Wireless Communications and Networking Conference (WCNC). IEEE, 2013, pp. 860-865.

[16] Y.-H. Yang, Y. Chen, C. Jiang, C.-Y. Wang, and K. R. Liu, "Wireless access network selection game with negative network externality," IEEE Transactions on Wireless Communications, vol. 12, no. 10, pp. 50485060, 2013.

[17] W. Ge, S. Chen, H. Ji, X. Li, and V. C. Leung, "Green access point selection for wireless local area networks enhanced by cognitive radio," Mobile Networks and Applications, vol. 18, no. 4, pp. 553-566, 2013.

[18] M. D. Jaraiz-Simon, J. A. Gomez-Pulido, and M. A. Vega-Rodriguez, "Embedded intelligence for fast qos-based vertical handoff in heterogeneous wireless access networks," Pervasive and Mobile Computing, vol. 19, pp. 141-155, 2015.

[19] M. H. Cheung, F. Hou, V. W. Wong, and J. Huang, "Dora: Dynamic optimal random access for vehicle-to-roadside communications," IEEE Journal on Selected Areas in Communications, vol. 30, no. 4, pp. 792 803, 2012

[20] G. Kousalya, P. Narayanasamy, J. H. Park, and T.-h. Kim, "Predictive handoff mechanism with real-time mobility tracking in a campus wide wireless network considering its," Computer Communications, vol. 31, no. 12 , pp. 2781-2789, 2008

[21] W. Wanalertlak, B. Lee, C. Yu, M. Kim, S.-M. Park, and W.-T. Kim, "Behavior-based mobility prediction for seamless handoffs in mobile wireless networks," Wireless Networks, vol. 17, no. 3, pp. 645-658, 2011.

[22] J. Kim, J.-D. Cho, J. Jeong, J.-Y. Choi, B.-h. Song, and H. Lee, "Fuzzy logic based handoff scheme for heterogeneous vehicular mobile networks," in International Conference on High Performance Computing \& Simulation (HPCS). IEEE, 2014, pp. 863-870.

[23] M. Tao, H. Yuan, S. Dong, and H. Yu, "Initiative movement prediction assisted adaptive handover trigger scheme in fast mipv6," Computer Communications, vol. 35, no. 10, pp. 1272-1282, 2012.

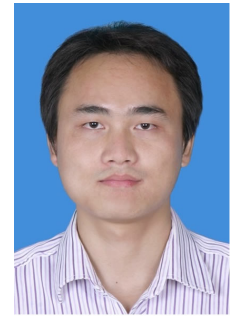

Ming Tao received his B.S. degree from Anhui University, China in 2007, and his M.S. and Ph.D. degrees from South China University of Technology (SCUT), China, in 2009 and 2012, respectively. He is currently an associate researcher at the School of Computer of Dongguan University of Technology, and the Director of the key laboratory of the wireless sensor network system of Dongguan. His primary research interests include protocol design and performance analysis in next-generation wireless/mobile networks, High Performance Computing and grid technology. He has served as a reviewer for several IEEE international conferences and International Journals.

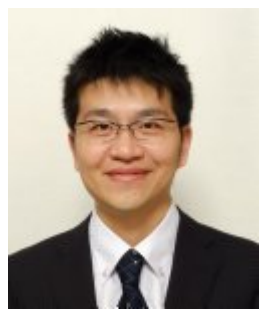

Mianxiong Dong received B.S., M.S. and Ph.D. in Computer Science and Engineering from The University of Aizu, Japan. He is currently an Associate Professor in the Department of Information and Electronic Engineering at the Muroran Institute of Technology, Japan. Prior to joining MuroranIT, he was a Researcher at the National Institute of Information and Communications Technology (NICT), Japan. He was a JSPS Research Fellow with School of Computer Science and Engineering, The University of Aizu, Japan and was a visiting scholar with BBCR group at University of Waterloo, Canada supported by JSPS Excellent Young Researcher Overseas Visit Program from April 2010 to August 2011. Dr. Dong was selected as a Foreigner Research Fellow (a total of 3 recipients all over Japan) by NEC C\&C Foundation in 2011. His research interests include Wireless Networks, Cloud Computing, and Cyber-physical Systems. His research results have been published in 130 research papers in international journals, conferences and books. He has received best paper awards from IEEE HPCC 2008, IEEE ICESS 2008, ICA3PP 2014, GPC 2015, and IEEE DASC 2015. Dr. Dong serves as an Editor for IEEE Communications Surveys and Tutorials, IEEE Network, IEEE Wireless Communications Letters, IEEE Cloud Computing, IEEE Access, and Cyber-Physical Systems (Taylor \& Francis), as well as a leading guest editor for ACM Transactions on Multimedia Computing, Communications and Applications (TOMM), IEEE Transactions on Emerging Topics in Computing (TETC), IEEE Transactions on Computational Social Systems (TCSS). He has been serving as Symposium Chair of IEEE GLOBECOM 2016, IEEE ICC 2017. Dr. Dong is currently a research scientist with A3 Foresight Program (2011-2016) funded by Japan Society for the Promotion of Sciences (JSPS), NSFC of China, and NRF of Korea.

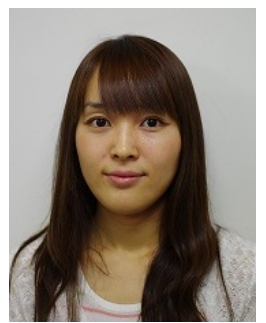

Kaoru Ota was born in Aizu Wakamatsu, Japan. She received M.S. degree in Computer Science from Oklahoma State University, USA in 2008, B.S. and $\mathrm{Ph} . \mathrm{D}$. degrees in Computer Science and Engineering from The University of Aizu, Japan in 2006, 2012, respectively. She is currently an Assistant Professor with Department of Information and Electronic Engineering, Muroran Institute of Technology, Japan. From March 2010 to March 2011, she was a visiting scholar at University of Waterloo, Canada. Also she was a Japan Society of the Promotion of Science (JSPS) research fellow with Kato-Nishiyama Lab at Graduate School of Information Sciences at Tohoku University, Japan from April 2012 to April 2013. Her research interests include Wireless Networks, Cloud Computing, and Cyber-physical Systems. Dr. Otas research results have been published in 110 research papers in international journals, conferences and books. She has received best paper awards from ICA3PP 2014, GPC 2015, and IEEE DASC 2015. She serves as an editor for Peer-to-Peer Networking and Applications (Springer), Ad Hoc \& Sensor Wireless Networks, International Journal of Embedded Systems (Inderscience), as well as a guest editor for IEEE Wireless Communications, IEICE Transactions on Information and Systems. She is currently a research scientist with A3 Foresight Program (2011-2016) funded by Japan Society for the Promotion of Sciences (JSPS), NSFC of China, and NRF of Korea.

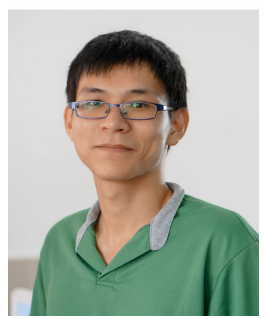

Zhimin He received his B.S. and Ph.D. degrees from the South China University of Technology, China, in 2010 and 2015, respectively. He is a lecturer at South China Agricultural University, Guangzhou, China. His current research interests include pattern recognition, adversarial learning, information security, and the Internet of Things. 